\title{
A Proteomic Approach for the Involvement of the GAPDH in Alzheimer Disease in the Blood of Moroccan FAD Cases
}

\author{
Nadia El Kadmiri • Raquel Cuardos • \\ Bouchra El Moutawakil • Ilham Slassi • Jesus Avila • \\ Sellama Nadifi • Ahmed Hachem • Abdelaziz Soukri
}

Received: 22 June 2014 / Accepted: 1 July 2014 /Published online: 15 July 2014

(C) Springer Science+Business Media New York 2014

\begin{abstract}
Several articles have highlighted the potential involvement of glyceraldehyde-3-phosphate dehydrogenase (GAPDH) in neurodegeneration by showing a nonglycolytic activity of GAPDH specifically in the brains of subjects with Alzheimer's disease (AD). The novel aim of this study was to elucidate the critical role of GAPDH and its interaction with $\beta$-amyloid in the blood of Moroccan patients with familial $\mathrm{AD}$ (FAD) carrying presenilin mutations and in sporadic late onset $\mathrm{AD}$ (LOAD). Our results show a significant decrease in the activity of GAPDH in blood samples from patients with FAD as compared to sporadic cases and healthy controls. The expression level of GAPDH in brain specimens from mutant tau transgenic mice and patients with FAD was unchanged as compared to healthy controls. In contrast, the expression level of GAPDH in blood samples from mutant tau transgenic mice and patients with FAD was decreased as compared to sporadic cases and healthy controls. Moreover,
\end{abstract}

N. El Kadmiri $(\bowtie) \cdot$ B. El Moutawakil $\cdot$ I. Slassi $\cdot$ S. Nadifi Laboratory of Medical Genetics and Molecular Pathology, Faculty of Medicine and Pharmacy, University Hassan II, 19 Rue Tarik Ibnou Ziad, B.P. 9154, Casablanca 20000, Morocco

e-mail: elkadmiri1979@gmail.com

N. El Kadmiri • A. Soukri

Laboratory of Physiology and Molecular Genetics, Faculty of

Sciences Aïn Chock, University Hassan II, Casablanca, Morocco

B. El Moutawakil • I. Slassi

Department of Neurology, CHU IBN ROCHD, Casablanca,

Morocco

R. Cuardos $\cdot$ J. Avila

CIBERNED - Investigations Center of Neurodegenerative

Diseases - the Centro de Biologia Molecular "Severo Ochoa",

Madrid, Spain

A. Hachem

Laboratory of Thrombosis and Hemostasis, Montreal Heart Institute,

Montreal, Quebec, Canada there is an accumulation of $\beta$-amyloid aggregates in the blood samples of patients with FAD and an increase in amyloid fibrils in both the blood and brain samples of these patients. Our study adds new insight to previous ones by showing the involvement of GAPDH in AD, which may influence the pathogenesis of this neurodegenerative disease.

Keywords Moroccan FAD · GAPDH

\section{Introduction}

Recent proteomic studies aimed at determining altered levels of a multitude of proteins in the brains of individuals with Alzheimer's disease (AD), which could be involved in $\mathrm{AD}$ pathogenesis (Butterfield et al. 2010). The identification of proteins affecting the degree of neurodegeneration could contribute to the development of biomarkers or new drug targets in the management of $\mathrm{AD}$.

$\mathrm{AD}$ is the most common neurodegenerative disease in the elderly, accounting for 50 to $56 \%$ of cases as assessed by autopsy and clinical series, with an annual incidence of approximately $3 \%$ in the 65-74 age group (Castellani et al. 2010). AD is clinically characterized by progressive impairment in memory and cognitive decline. In Morocco, AD affects almost 30,000 individuals, and this number could increase to 75,000 by 2020 (projections of the World Health Organization (WHO)). However, this disease remains poorly understood by the Moroccan general public. To our knowledge, the genes predisposing individuals to $\mathrm{AD}$ and predicting disease incidence remain elusive, and no proteomic studies have been carried out in this area. AD is pathologically characterized by extracellular deposition of $\beta$-amyloid $(A \beta)$ in senile plaques and formation of intracellular neurofibrillar tangles, mainly composed of the hyperphosphorylated microtubule-associated protein tau (Ling et al. 2003; 
Gomez-Ramos et al. 2004). Several articles have highlighted the potential involvement of glyceraldehyde-3-phosphate dehydrogenase (GAPDH; EC 1.2.1.12) in neurodegeneration by reporting a non-glycolytic activity of GAPDH specifically in brains of AD cases. GAPDH strongly interacts with the $\beta$ amyloid precursor protein ( $\beta$-APP) with high affinity in vitro and it is found as a protein component of neurofibrillary tangles (NTFs). Whether GAPDH is also involved in the initiation of programmed cell death cascades is still unknown (Ling et al. 2003; Schulze et al. 1993; Chen et al. 2000; Oyama et al. 2000; Mazzola and Sirover 2001; Cumming and Schubert 2005; Verdier et al. 2005; Nakajima et al. 2007; Naletova et al. 2008).

The aim of this study is to probe for the role of GAPDH and its interaction with $\mathrm{A} \beta$ amyloid aggregates especially in blood samples from Moroccan patient of FAD cases carrying frameshift mutations in the presenilin genes.

\section{Methods}

Sample Preparation

\section{Human Blood}

Peripheral blood was taken from three FAD cases (one woman (ID: F1) with a disease onset age of 63 years and two men with disease onset age of 60 (ID: F2) and 55 (ID: F3)years respectively), 17 sporadic cases (7 men and 10 women; age $66 \pm$ 10,81 ), and two healthy controls (two women aged 58 and 55 years). All patients were seen by the Memory Consultation group at the CHU IBN ROCHD Neurology Department in Casablanca, Morocco. The protocol was approved by the Human Ethical Committee of the CHU IBN ROCHD in accordance with the Declaration of Helsinki for experiments involving humans, and written consent was obtained from the patients and their legal guardians prior to their enrollment in the study. A family history was obtained by a clinical interview of the patient and a "yes" or "no" self-reporting questionnaire from the legal guardian or other family member. The disease was considered familial if at least one additional first degree relative suffered from early onset AD-type dementia. All patients underwent standard somatic neurological examination, cognitive function assessment, brain imaging, and laboratory tests. The clinical, neuropathological, and genetic assessments of FAD cases have several shared features. For instance, there is a strong correlation between clinical symptoms and genetic factors in our patients with a family history of AD. Our mutational analysis (El Kadmiri et al. 2014) revealed that our FAD cases carry frameshift mutations located in the presenilin genes (F1/exon 11/PS1, F2/exon 5/PS2, and F3/Exon 9/PS2 respectively), whereas our sporadic cases do not carry mutations in the presenilin genes.

\section{Human Brain Samples}

Brain specimens were removed at autopsy from one patient with confirmed FAD and one healthy control. Brain specimens were frozen and stored at $-80^{\circ} \mathrm{C}$ at CIBERNEDInvestigations Center of Neurodegenerative Diseases - the Centro de Biologia Molecular "Severo Ochoa" (Madrid, Spain). Written informed consents from the donors and their next of kin were obtained for using the brain samples in research in accordance with approved protocols by the local Ethical Committees of the CIBERNED - the Centro de Biología Molecular "Severo Ochoa" brain banks for nervous tissue donation.

\section{Animals}

Mutant tau transgenic mice were engineered, bred, and housed under pathogen-free conditions at the CIBERNED animal facility. Their wild type (WT) littermates were used as controls. Handling and caring of mice were in compliance with the guidelines established by the Animal Care and Ethical Committee of CIBERNED. Only the male mice were used for this study.

Mouse Blood Retrieval Murine blood was retrieved by cardiac puncture from WT and mutant tau transgenic-anesthetized mice into $1 \mathrm{~mL}$ syringes containing $50 \mu \mathrm{L}$ of heparin $(1,000 \mathrm{U} / \mathrm{mL})$.

Mouse Brain Mice were sacrificed with an overdose of anesthetics to minimize suffering. After dissection, the brains from WT and mutant tau transgenic mice were quickly removed. The brains were then dissected on an ice-cold plate, and brain samples were placed in tubes on ice for immediate use.

\section{Blood Protein Extraction}

Protein extracts were obtained from red blood cells (RBC). Briefly, blood samples (human or mouse) were centrifuged, and the RBC layer was recovered after discarding the plateletrich plasma and white blood cell layers. The RBC extracts were washed with isotonic $\mathrm{NaCl}(0.9 \%)$ and then lysed with ice-cold lysis buffer (Hepes $20 \mathrm{mM}$ ph 7.4, NaCl $100 \mathrm{mM}$, orthovanadate $1 \mathrm{mM}$, EDTA $5 \mathrm{mM}, 1 \%$ Triton X-100, $1 \times$ protease inhibitor cocktail and okadaic acid $100 \mu \mathrm{M})$.

\section{Brain Protein Extraction}

Brain samples were homogenized four times for $25 \mathrm{~s}$, centrifuged for $1 \mathrm{~h}$ at 10,000 RPM, and lysed with the abovementioned lysis buffer. Brain protein extracts were then recovered after centrifugation for $30 \mathrm{~min}$ at 22,000 RPM. 


\section{GAPDH Activity Assay}

The phosphorylating activity of GAPDH was determined. The reaction was started by the addition of $10 \mu \mathrm{g}$ of protein preparation to an assay mixture containing $50 \mathrm{mM}$ Tricine buffer ( $\mathrm{pH} 8.5), 10 \mathrm{mM}$ sodium arsenate, $1 \mathrm{mM} \mathrm{NAD}^{+}$, and $2 \mathrm{mM}$ D-glyceraldehyde-3-phosphate (D-G3P). The total volume of the reaction mixture was $1 \mathrm{ml}$. Absorbance changes at $340 \mathrm{~nm}$ were monitored. One minute of enzyme activity was defined as the amount of enzyme that catalyzes the reduction of $1 \mu \mathrm{mol} \mathrm{NAD}{ }^{+}$per minute. All experiments and assays were carried out in triplicates.

\section{GAPDH Protein Expression}

The protein samples were assayed for their protein concentration via Bradford. The equivalent of 30-40 $\mu \mathrm{g}$ of proteins was mixed with Laemmli sample buffer, boiled for $3 \mathrm{~min}$, resolved in $10 \%$ SDS-PAGE gels, and transferred onto nitrocellulose membranes (Schleicher and Schuell, Dassel, Germany). The membranes were blocked with $5 \%$ non-fat dry milk for $1 \mathrm{~h}$, washed three times with PBS/Tween $(150 \mathrm{mM} \mathrm{NaCl}, 20 \mathrm{mM}$ Tris, $\mathrm{pH}$ 7.4, $0.1 \%$ Tween-20), and incubated $1 \mathrm{~h}$ at room temperature with the mouse monoclonal anti-GAPDH antibody (1:5,000 dilution) (Abcam, Spain). Following the washing steps, membranes were labeled with horseradish peroxidase (HRP)-conjugated secondary antibody (goat anti-Mouse IgG HRP-conjugated, Santa Cruz biotechnology, Spain) for $1 \mathrm{~h}$; washed and bound peroxidase activity was then detected by the enhanced chemiluminescence detection system (Amersham Biosciences, Arlington Heights, IL). To asses equal amounts of protein loading, membranes were stripped, blocked with $5 \%$ milk, and blotted for $\beta$-actin $(1: 2,000$ dilution) (mouse monoclonal anti- $\beta$-actin antibody, SigmaAldrich, Spain). Protein bands were analyzed with an imaging densitometer.

\section{$\beta$-Amyloid Protein Aggregates}

A $\beta$ protein SC489 and its monoclonal HRP-conjugated antibody 4G8 were purchased from Covance. One microliter of A $\beta$ protein SC489 was added per $10 \mu \mathrm{g}$ of protein extracts. All samples were heated for $30 \mathrm{~min}$ at $37^{\circ} \mathrm{C}$. The samples were deposited onto nitrocellulose membranes (Schleicher and Schuell, Dassel, Germany). The membranes were blocked with $5 \%$ non-fat dry milk at room temperature for $30 \mathrm{~min}$, washed three times with PBS/Tween, and incubated for $1 \mathrm{~h}$ at room temperature with the 4G8 antibody followed by another $1 \mathrm{~h}$ of incubation with an HRP-conjugated IgG. Washed and bound peroxidase activity was then detected using the ImmobilonTM Western chemiluminescent HRP substrate system. Spots were manually selected, and intensities of the spots were analyzed as mean gray values corrected for the background value using Image J software (Abramoff et al. 2004).

\section{EM}

One microliter of $\mathrm{A} \beta$ protein was added per $10 \mu \mathrm{g}$ of protein extracts. All samples were heated for $30 \mathrm{~min}$ at $37^{\circ} \mathrm{C}$. The samples were then stained with $2 \%$ uranyl acetate for $1 \mathrm{~min}$ followed by electron microscopy (EM) imaging.

\section{Results}

We first sought to evaluate the activity of GAPDH in the blood and brain of FAD cases - the blood from sporadic cases and healthy individuals. As shown in Fig. 1, the activity of GAPDH in protein extracts from both the peripheral blood and brain specimens from Moroccan patients with FAD was significantly decreased as compared to that of healthy controls. The activity of GAPDH in protein extracts from peripheral blood from Moroccan sporadic cases was not altered (data not shown). This decrease in activity was evaluated as a loss of absorbance, which was present throughout the 1-min time interval of incubation of the protein extracts from the FAD samples with the assay mixture. To evaluate the relationship between the expression and the activity of GAPDH in the samples, the protein extracts were assayed for their content in GAPDH. As shown in Fig. 2, the expression of GAPDH in the brain specimens from mutant tau transgenic mice and FAD case was unchanged compared to the healthy controls. In contrast, the expression of GAPDH in protein extracts form the peripheral blood of mutant tau transgenic mice and FAD cases was decreased as compared to controls. The expression of GAPDH in protein extracts form the peripheral blood from sporadic cases was non-significant (data not shown).

To investigate the effect of the frameshift mutations on the structural properties of $A \beta$ and the influence of GAPDH on $A \beta$ protein aggregates formation, protein extracts from healthy subjects and individuals with FAD were assayed for their potential to induce $A \beta$ aggregates and analyzed for the presence of amyloid fibrils. Interestingly, incubation of peripheral blood protein extracts with $A \beta$ induces an increase in $\mathrm{A} \beta$ accumulation in samples from FAD cases but not from healthy controls (Fig. 3). These FAD cases each carry a single frameshift mutation. The F1 case has a frameshift mutation in $73683837-\mathrm{G}$ at codon 378 , which is caused by a singlenucleotide insertion located in exon 11 of PS1, whereas the F2 case has a frameshift mutation in 227073260-A, which is caused by a single-nucleotide insertion at codon 126 located in exon 5 of PS2 (El Kadmiri et al. 2014). Furthermore, EM examination of the protein extracts and $A \beta$ incubations showed significant amyloid fibrils formation with brain 
Fig. 1 Activity assay of GAPDH in protein extract from the peripheral blood and brain. Histogram represents the absorbance of the protein sample at $340 \mathrm{~nm}$ over the $1-\mathrm{min}$ incubation period. $F 1$ and $F 2$ are protein extracts from the peripheral blood from two FAD cases, $B C$ protein extracts from brain specimens of a healthy control obtained at autopsy, $B A D$ protein extracts from brain specimens of a confirmed $\mathrm{AD}$ case at autopsy, and $C$ protein extracts from peripheral blood from a healthy subject

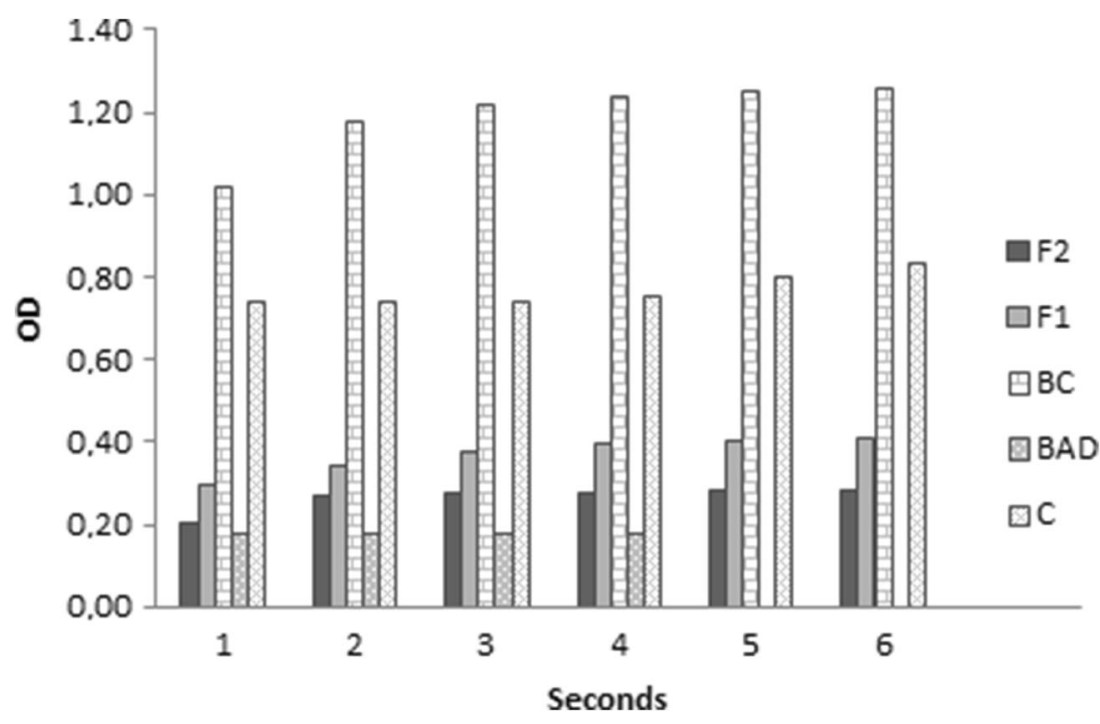

extract from a confirmed $\mathrm{AD}$ case and from peripheral blood of our FAD cases, which carries the frameshift mutation. Conversely, no formation of amyloid fibrils was observed when protein extracts from sporadic cases (data not shown) or healthy subjects was used (Fig. 4).

\section{Discussion}

Our finding shows a significant decrease in GAPDH activity albeit unchanged protein expression in the brain of both mutant tau transgenic mice and FAD case, which may be due to post-translational alterations. This result is consistent with previous studies demonstrating a decrease in GAPDH glycolytic activity regardless of the change in its expression in brain specimens of AD cases (Schulze et al. 1993; Mazzola and Sirover 2001; Cumming and Schubert 2005). In contrast, the expression of GAPDH in the blood of mutant tau transgenic mice and our FAD cases was decreased in comparison to controls, probably due to an alteration at the transcriptional level.

Several studies have confirmed the crucial role of GAPDH in influencing amyloid aggregation through post-translational alterations leading to a significant reduction in amyloid activity. These studies focused on the molecular mechanism by which GAPDH influences in amyloid aggregation. Moreover, GAPDH has previously been shown to bind to the cytoplasmic C-terminal domain of the amyloid precursor protein (APP) (Schulze et al. 1993). Recent studies confirmed that GAPDH could also bind a variety of $A \beta$ isoforms, and it displays a greater affinity for the A $\beta 1-42$ form (Cumming and Schubert 2005; Verdier et al. 2005; Naletova et al. 2008). Indeed, GAPDH is locally deposited in aggregates at lesion sites in $\mathrm{AD}$ via the formation of intermolecular disulfide bonds. Several studies have demonstrated that only the oxidized and denatured forms of GAPDH are able to form highly stable complexes with $A \beta$, indicating the direct involvement of GAPDH in amyloid aggregation. Cumming and Schubert 2005 revealed an increase in GAPDH intermolecular disulfide

\begin{tabular}{|c|c|c|c|c|c|c|c|c|c|c|}
\hline & $\begin{array}{c}\text { Brain } \\
\text { specimen } \\
\text { of } A D\end{array}$ & $\begin{array}{c}\text { Brain } \\
\text { specimen } \\
\text { of healthy } \\
\text { control }\end{array}$ & $\begin{array}{c}\text { Brain } \\
\text { specimen } \\
\text { of Mutant } \\
\text { tau } \\
\text { transgenic } \\
\text { mice }\end{array}$ & $\begin{array}{l}\text { Brain } \\
\text { specimen } \\
\text { of Wild } \\
\text { type mice }\end{array}$ & $\begin{array}{c}\text { Blood of Mutant } \\
\text { tau transgenic } \\
\text { mice }\end{array}$ & $\begin{array}{l}\text { Blood of Wild } \\
\text { type mice }\end{array}$ & $\begin{array}{c}\text { Blood } \\
\text { of healthy } \\
\text { subject }\end{array}$ & $\begin{array}{c}\text { Blood } \\
\text { of FAD (F3) }\end{array}$ & $\begin{array}{c}\text { Blood } \\
\text { of FAD (F2) }\end{array}$ & $\begin{array}{c}\text { Blood } \\
\text { of FAD (F1) }\end{array}$ \\
\hline \\
\hline (42 kDa) & & & & & & & & & & \\
\hline GAPDH & & & & & & & & & 5 & \\
\hline Expression & & & & & & & & & & \\
\hline$(37 \mathrm{kDa})$ & & & & & & & & & & \\
\hline
\end{tabular}

Fig. 2 Representative Western blots for GAPDH performed on protein extracts from the indicated samples. Blots for $\beta$-actin are from stripped GAPDH membranes 


\section{$\begin{array}{llll}\text { C1 } & \text { C2 } & \text { F2 } & \text { F1 }\end{array}$}

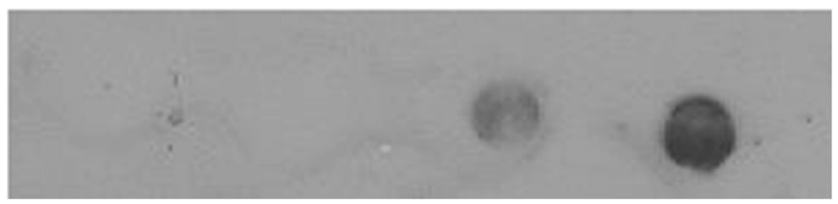

Fig. 3 Representative dot blot of formed A $\beta$ aggregates. Protein extracts from peripheral blood from two FAD cases (F1 and F2) and two control individuals $(\mathrm{C} 1$ and $\mathrm{C} 2)$ were incubated with $\mathrm{A} \beta$ and then transferred onto nitrocellulose membranes. Membrane were then blotted for $\mathrm{A} \beta$ to assess the potential of the protein extracts to induce accumulation of $A \beta$ aggregates

bonding in all examined $\mathrm{AD}$ brain extracts as compared to age-matched controls. The authors also verified the activity of GAPDH and showed that the oxidant-induced disulfide bonding of GAPDH leads to a reduction in its enzymatic activity. On the other hand, tau has been shown to bind and promote the denaturation and inactivation of GAPDH in vitro, suggesting a direct involvement of GAPDH in tau aggregation and NFT formation in brains of individuals with AD (Chen et al. 2000; Wang et al. 2005; Yang et al. 2008).

All these findings are consistent with our proteomic results, which assess the aggregation of amyloid beta in patients carrying mutations located in presenilin genes. Dot blotting showed increased $A \beta$ accumulation in peripheral blood from our FAD cases but not in healthy controls. EM examination revealed significant amyloid fibril formation both in brain specimens from confirmed $\mathrm{AD}$ case and in peripheral blood from our FAD cases, who carry frameshift mutations located in presenilin genes. Based on our results and results from other studies, we can conclude that there is a link between amyloid aggregation and GAPDH through conversion of the normal conformation of GAPDH to an abnormal one, which leads to a decrease in its activity. Whether GAPDH is also involved in the initiation of the programmed cell death cascades remains to be determined. Moreover, ROS have been shown to play an important role in modulating the non-glycolytic functions of GAPDH, which may significantly contribute to loss of neuronal function and neurodegeneration in brains of $\mathrm{AD}$ patients (Butterfield and Lauderback 2002; Uttara et al. 2009).

Our mutational and proteomic analysis report a correlation between genotype and phenotype in our cases of EOAD involving the frameshift presenilin mutations and suggest that these mutations increase the risk of developing neurodegenerative diseases through $A \beta-42$ accumulation, which is due to modulation in the proteolytic processing of APP (De Strooper et al. 2012). This modulation is manifested by beta amyloid aggregates that directly interact with GAPDH and alter its function.

Identification of proteins affecting the degree of neurodegeneration could contribute to the development of biomarkers or new drug targets in the management of AD. Cerebrospinal fluid (CSF) and blood plasma are the most promising sources for AD biomarkers as compared to brain tissue. These fluids are more easily accessible, which was the aim of this study, differentiating it from several others ones to elucidate the critical role of GAPDH in the blood of AD individuals that may influence the pathogenesis of neurodegenerative diseases. However, it remains to clarify this decreased expression of GAPDH observed in the blood compared to brain. A study
Fig. 4 Representative electron microscopy images of formed $\mathrm{A} \beta$ aggregates. Protein extracts from a protein extracts from brain specimens of a confirmed $\mathrm{AD}$ case at autopsy, $\mathbf{b}$ protein extracts of peripheral blood from a FAD case (ID. F1), c protein extracts from the peripheral blood from a FAD case (ID.F2), $\mathbf{d}$ protein extracts from brain specimens from a healthy subject at autopsy, e protein extracts from peripheral blood from a healthy subject, and f PBS as control were incubated with $A \beta$ and then visualized on an electron microscopic to asses amyloid fibril formation. $\mathbf{a}, \mathbf{b}$, and c show significant amyloid fibril formation, whereas $\mathbf{d}, \mathbf{e}$, and $\mathbf{f}$ show no abnormalities. The scale bar for images $\mathbf{a}, \mathbf{b}, \mathbf{c}$, and $\mathbf{d}$ represents $70 \mathrm{~nm}$, whereas that for images $\mathbf{e}$ and $\mathbf{f}$ represents $100 \mathrm{~nm}$
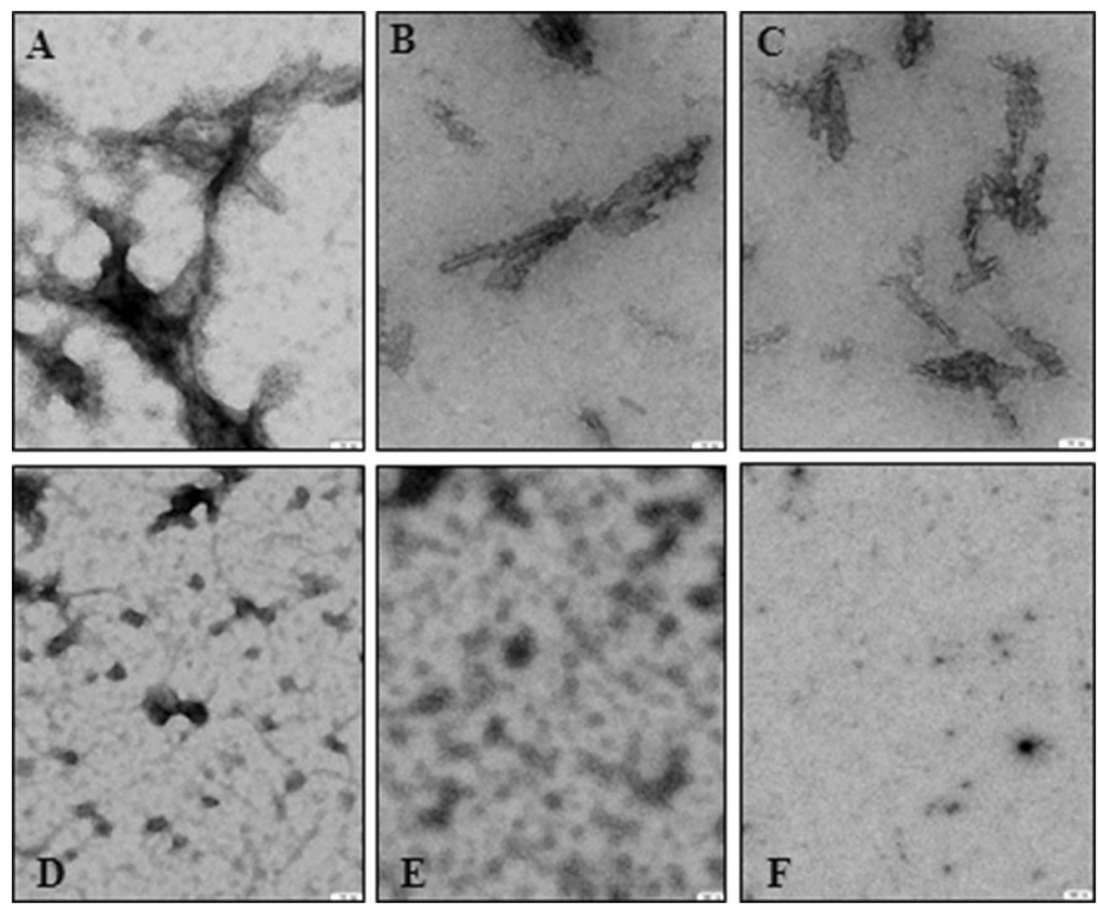
is underway to eventually demonstrate the mechanism responsible for this decreased expression and its involvement in the neurodegenerative process of AD. These findings open prospects for diagnosis by using GAPDH as a biomarker for diagnostics and monitoring AD modification.

Acknowledgments We would like to thank Ranbanxy Morocco LCC for their partial financial support to Miss Nadia El Kadmiri. We thank Mr. Othman Rouissi at Ranbanxy Morocco LLC and the staff at the CHU IBN ROCHD Neurology Department in Casablanca for their technical assistance. We thank all the staff at CIBERNED - Investigations Center of Neurodegenerative Diseases - the Centro de Biologia Molecular "Severo Ochoa" (Madrid, Spain) for their technical assistance. We also thank the staff at the Medical Genetics Institute at the Universita Catholica in Rome, Italy, for their help in gene sequencing. We thank Dr. B. Elkhalfi at the LPGM for her help.

Conflict of interest The authors declare that no competing interests exist.

Funding The funders had no role in study design, data collection and analysis, decision to publish, or preparation of the manuscript. No additional external funding was received for this study.

\section{References}

Abramoff MD, Magalhaes PJ, Ram SJ (2004) Image processing with image. J Biophoton Int 11:36-42

Butterfield DA, Lauderback CM (2002) Lipid peroxidation and protein oxidation in Alzheimer's disease brain: potential causes and consequences involving amyloid beta-peptide-associated free radical oxidative stress. Free Radic Biol Med 32:1050-1060

Butterfield DA, Hardas SS, Lange ML (2010) Oxidatively modified glyceraldehyde-3- phosphate dehydrogenase (GAPDH) and Alzheimer's Disease: many pathways to neurodegeneration. J Alzheimers Dis 20:369-393

Castellani RJ, Rolston RK, Smith MA (2010) Alzheimer disease. Dis Mon 56:484-546

Chen YH, He RQ, Liu Y, Xue ZG (2000) Effect of human neuronal tau on denaturation and reactivation of rabbit muscle D-glyceraldehyde-3phosphate dehydrogenase. Biochem J 351:233-240
Cumming RC, Schubert D (2005) Amyloid beta induces disulfide bonding and aggregation of GAPDH in Alzheimer's disease. FASEB J 19:2060-2062

De Strooper B, Iwatsubo T, Wolfe MS (2012) Presenilins and $\gamma$-secretase: structure, function, and role in Alzheimer disease. Cold Spring Harb Perspect Med 2:a006304

El Kadmiri N, Zaid N, Zaid Y, Tadevosyan A, Hachem A, Dubé MP, Hamzi K, El Moutawakil B, Slassi I, Nadifi S (2014) Novel presenilin mutations within Moroccan patients with early-onset Alzheimer's disease. Neuroscience 269:215-222

Gomez-Ramos A, Smith MA, Perry G, Avila J (2004) Tauphosphorylation and assembly. Acta Neurobiol Exp (Wars) 64:33-39

Ling Y, Morgan K, Kalsheker N (2003) Amyloid precursor protein (APP) and the biology of proteolytic processing: relevance to Alzheimer's disease. Int J Bioch Cell Biol 35:1505-1535

Mazzola JL, Sirover MA (2001) Reduction of glyceraldehyde-3phosphate dehydrogenase activity in Alzheimer's disease and in Huntington's disease fibroblasts. J Neurochem 76:442-449

Nakajima H, Amano W, Fujita A et al (2007) The active site cysteine of the proapoptotic protein glyceraldehyde-3-phosphate dehydrogenase is essential in oxidative stress-induced aggregation and cell death. J Biol Chem 282:26562-26574

Naletova I, Schmalhausen E, Kharitonov A et al (2008) Non-native glyceraldehyde-3-phosphate dehydrogenase can be an intrinsic component of amyloid structures. Biochim Biophys Acta 1784:2052-2058

Oyama R, Yamamoto H, Titani K (2000) Glutamine synthetase, hemoglobin a-chain, and macrophage migration inhibitory factor binding to amyloid b-protein: their identification in rat brain by a novel affinity chromatography and in Alzheimer's disease brain by immunoprecipitation. Biochim Biophys Acta 1479:91-102

Schulze H, Schuler A, Stuber D et al (1993) Rat brain glyceraldehyde-3phosphate dehydrogenase interacts with the recombinant cytoplasmic domain of Alzheimer's B-amyloid precursor protein. J Neurochem 60:1915-1922

Uttara B, Singh AV, Zamboni P, Mahajan RT (2009) Oxidative stress and neurodegenerative diseases: a review of upstream and downstream antioxidant therapeutic options. Curr Neuropharmacol 7:65-74

Verdier Y, Huszar E, Penke B et al (2005) Identification of synaptic plasma membrane proteins co-precipitated with fibrillar b-amyloid peptide. J Neurochem 94:617-628

Wang Q, Woltjer RL, Cimino PJ et al (2005) Proteomic analysis of neurofibrillary tangles in Alzheimer disease identifies GAPDH as a detergent-insoluble paired helical filament tau binding protein. FASEB J 19:869-871

Yang G, Wang L, Zhu M, Xu D (2008) Identification of non-Alzheimer's disease tauopathies-related proteins by proteomic analysis. Neurol Res 30:613-622 